\title{
Cómo afrontar la renovación de la anatomía artística en Bellas Artes. Proyecto de innovación docente en la asignatura de "Anatomía y Morfología".
}

\section{How to face the renewal of artistic anatomy in Fine Arts. Teaching innovation project in the subject of "Anatomy and Morphology".}

Jesús Algovi GonZÁleZ VILLEgAS.

Departamento de Dibujo.

Facultad de Bellas Artes de la

Universidad de Sevilla.

Email: jesusalgovi@us.es

ORCID ID: https://orcid.org/0000-0003-1986-6771

DOI: http://dx.doi.org/10.12795/9788447231003.032

Pp.: 671-694 


\section{Introducción. Breve descripción del contexto.}

Para la aplicación del ciclo de mejora en el aula (CIMA), dentro del curso FIDOP 2020, he elegido una materia que imparto en el primer cuatrimestre. Se trata de la asignatura denominada Anatomía y Morfología, obligatoria y correspondiente al Departamento de Dibujo de la Facultad de Bellas Artes de la Universidad de Sevilla, al que pertenezco como miembro docente (PSI) desde 2010. La asignatura de Anatomía y Morfología, a la que corresponden 6 créditos, se imparte en el primer cuatrimestre, dentro del 2o curso de Grado en Bellas Artes. Es de una asignatura de 4 horas semanales de docencia, repartidas en dos días de clase semanales, a la que se suman (con un alumno interno) 3 horas de taller (90 minutos de talles posteriores a cada clase). Están 27 alumnos matriculados en el grupo 4 e igualmente 27 matriculados en el grupo 6, de los cuales soy profesor único el cuatrimestre completo.

\section{Metodología. Aspectos iniciales para la mejora docente.}

Dado el reto planteado dentro de este curso de innovación docente, elaboré algunos aspectos y herramientas pedagógicas que pudiese introducir o implementar durante la aplicación de este ciclo de mejora en el aula:

\section{Mejora de la motivación}

- Finalidad de la actividad. Incentivar mediante la asunción de esfuerzo-resultado.

- Repercusión en su futuro profesional. Posibilidad de trascender mediante el ejercicio.

- Mejora de las habilidades en red. Ciclos de Mejora en el Aula (2020). Experiencias de Innovación Docente de la US
Esta obra se distribuye con la licencia Creative Commons 
- Reelaboración del problema, mediante la inclusión de la opinión de los estudiantes (encuesta en clase, preguntas abiertas, etc).

- Estudio de caso, real con aplicación práctica y desarrollo de su imaginación creativa.

\section{Mejora de la atención}

- Efecto "sorpresa". Introducir elementos para ello que rompan con su rutina.

- Introducción de actividades novedosas (aula invertida / flipped classroom).

- Cambio de entorno.

- Trabajo en grupo.

\section{Mejora de la comunicación}

- Lenguaje asequible para el estudiante. Evitar el lenguaje excesivamente científico.

- Enseñar los códigos de comunicación específicos y el vocabulario científico.

- Utilizar medios cercanos o familiares en el entorno de intereses de los alumnos.

- Utilización de la tecnología (TIC). Plataforma de Enseñanza Virtual:creación de blog como foro de debate interno y autónomo. Probar una actividad interactiva.

- Bibliografía y debate abierto en clase sobre ella.

\section{Diseño metodológico}

En base a lo anteriormente planteado y a lo asimilado en este curso de formación, propuse como planificación metodológica para su aplicación en este ciclo de mejora en el aula, el siguiente esquema del modelo metodológico posible.

Ciclos de Mejora en el Aula (2020). Experiencias de Innovación Docente de la US Esta obra se distribuye con la licencia Creative Commons 


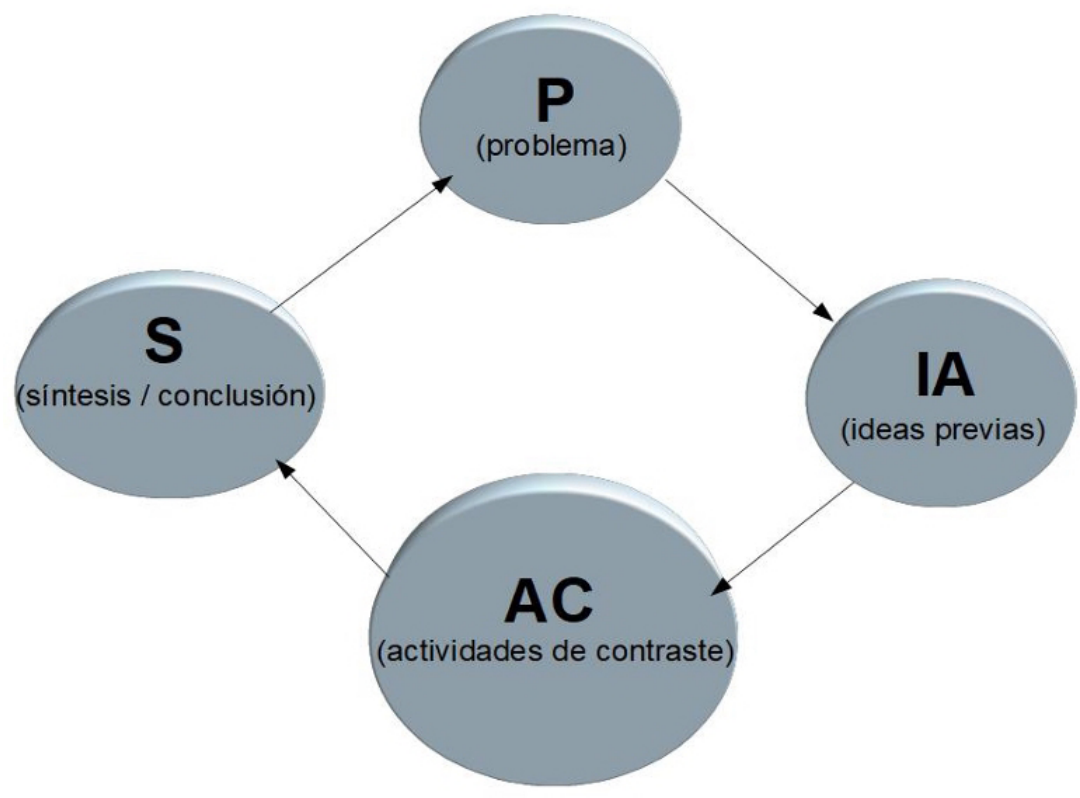

Figura 1. Modelo metodológico

Un segundo esquema aplicado al taller conceptual/laboratorio creativo:

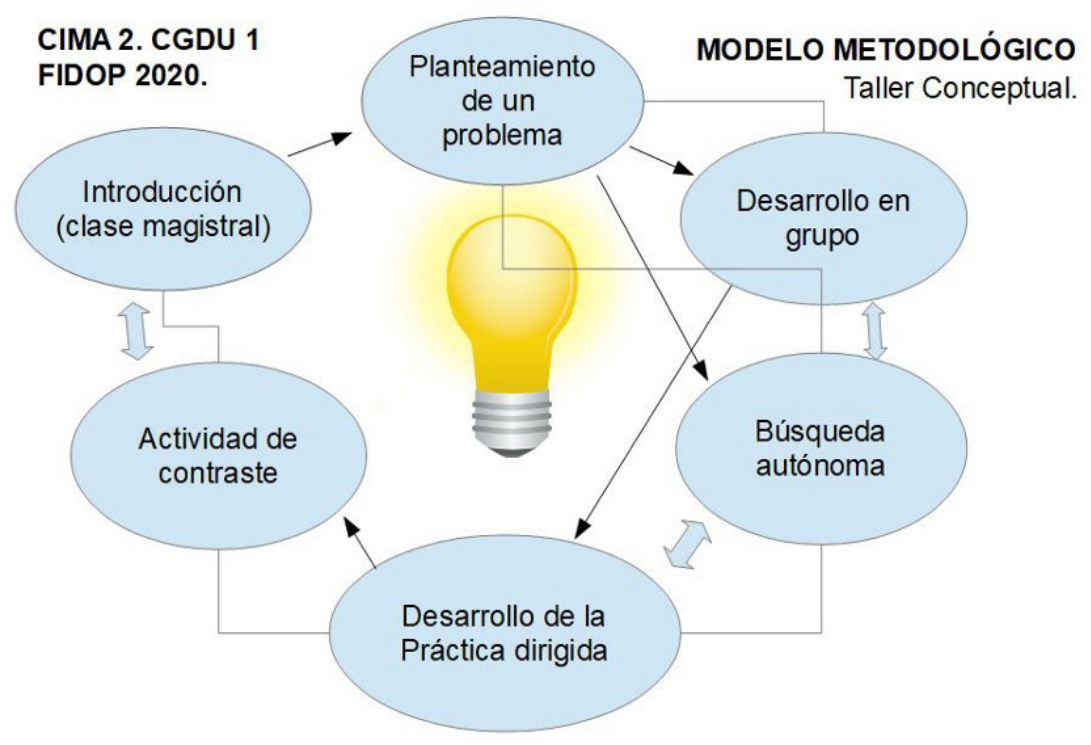

Figura 2. Modelo metodológico del taller conceptual

Ciclos de Mejora en el Aula (2020). Experiencias de Innovación Docente de la US (c) (1) E Esta obra se distribuye con la licencia Creative Commons Reconocimiento-NoComercial-SinObraDerivada Internacional (CC BY-NC-ND 4.0.) 


\section{Diseño. Planificación previa del ciclo de mejora en el aula (CIMA).}

\section{Cronograma previo publicado.}

Al iniciar este curso de FIDOP 2020, ya tenía publicado el cronograma de la asignatura completa en la Plataforma de Enseñanza Virtual. Por tanto el CIMA lo debía aplicar en la segunda y tercera prácticas evaluables dentro del programa de la asignatura. Esto condicionaba este ciclo respecto a la posibilidad de revisar el mapa de contenidos. Estas prácticas engloban la totalidad de dos semanas. Este es el segundo año que la imparto esta asignatura completa. Debía intentar planificar e introducir mejoras a la misma, aplicando los conocimientos asimilados.

Mapa de contenidos y problemas.

En base a los mencionado anteriormente, elaboré este mapa de contenidos para el ciclo:

Ciclos de Mejora en el Aula (2020). Experiencias de Innovación Docente de la US Esta obra se distribuye con la licencia Creative Commons 

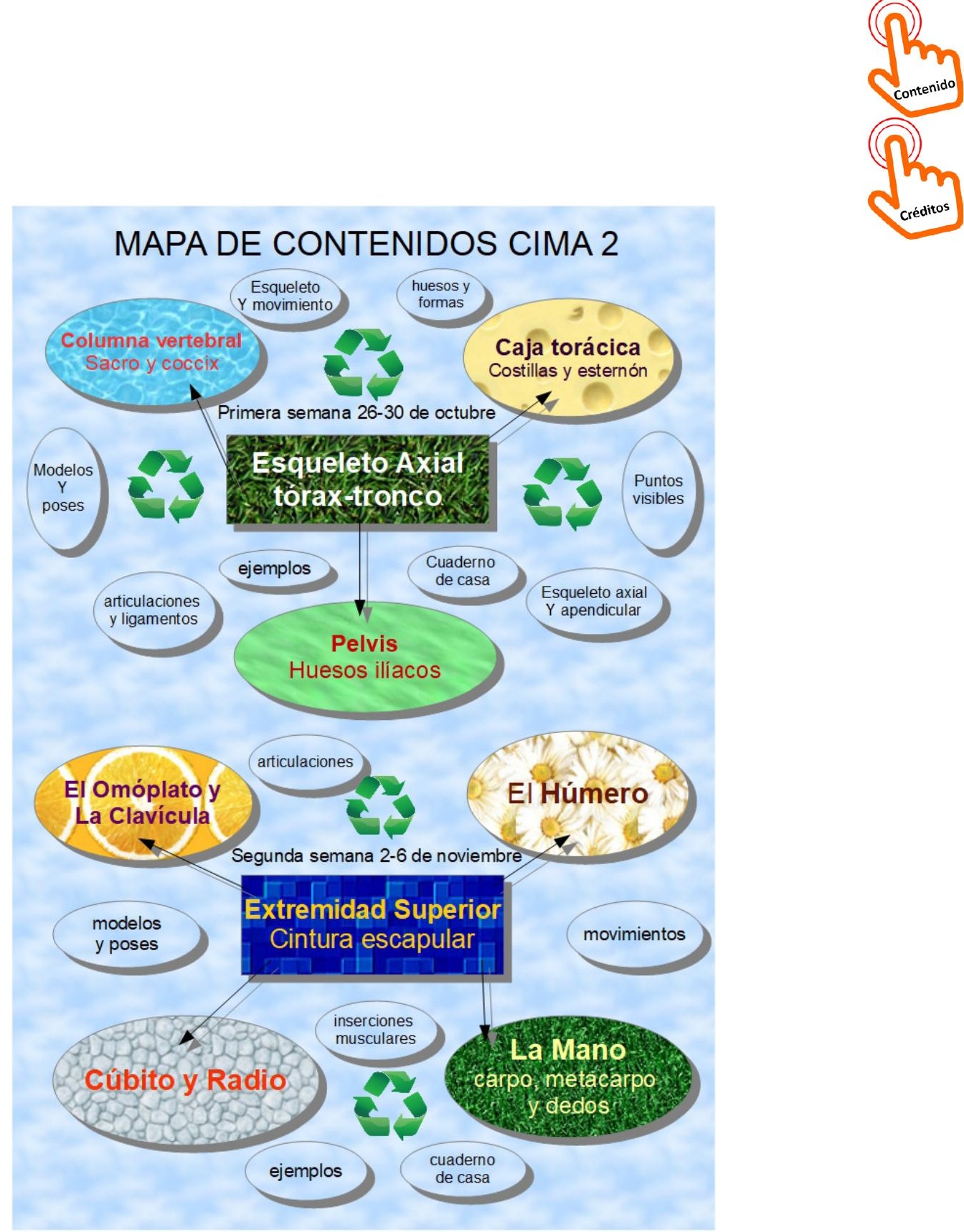

Figura 3. Mapa de contenidos y problemas.

Ciclos de Mejora en el Aula (2020). Experiencias de Innovación Docente de la US (C) (i) $\odot$ Esta obra se distribuye con la licencia Creative Commons Internacional (CC BY-NC-ND 4.0.) 


\section{Tabla / cronograma. Secuencia de actividades del ciclo de mejora.}

En consonancia con lo mencionado en los apartados anteriores, puse en práctica la metodología y los contenidos elaborando la siguiente secuencia de actividades para las dos semanas previstas:

Tabla 1. Secuencia de actividades del ciclo de mejora

\begin{tabular}{|c|c|c|}
\hline \multicolumn{3}{|c|}{$\begin{array}{l}\text { Sesión 1: Planteamiento del problema, cuestionario inicial, introducción } \\
\text { teórica (inicio clásico, en la 1â semana) y práctica de taller conceptual. } \\
\text { Grupo } 6 \text {. Anatomía y Morfología. Facultad de Bellas Artes. US. }\end{array}$} \\
\hline Modelo & \begin{tabular}{|l|l|} 
№ 1 $(28 / 10 / 2020)$ & $\begin{array}{l}\text { Planteamiento del } \\
\text { problema }\end{array}$
\end{tabular} & empo \\
\hline$A C$ & \multicolumn{2}{|c|}{$\begin{array}{l}\text { Comienzo con la presentación del CIMA 2. Les explico el sentido del } \\
\text { cuestionario (anónimo, en dos fases, referenciado y sin calificación). } \\
\text { Les entrego y rellenan el cuestionario (10 minutos). } \\
\text { Después les entrego la fotocopia (anexo) con la práctica del taller } \\
\text { conceptual I laboratorio creativo, para que vayan leyendo las } 4 \\
\text { preguntas que la componen. } \\
\text { Comienzo con el planteamiento del problema: la representación } \\
\text { gráfica y reconocimiento morfológico sobre los modelos desnudos } \\
\text { (dibujo del natural) de la Caja torácica (costillas y esternón) y de la } \\
\text { cintura pelviana (huesos iliacos, sacro y cóccix). } \\
\text { Introducción teórica (T), con clase magistral (ppt / audiovisual). Les } \\
\text { planteo multitud de ejemplos y contraejemplos tanto en el arte } \\
\text { contemporáneo como en el ámbito cotidiano. }\end{array}$} \\
\hline$A C$ & $\begin{array}{l}\text { entienden ellos por dibujo?. ¿para } \\
\text { y cómo nos afecta en el dibujo?. } \\
\text { futuro profesional?. ¿Qué es el es } \\
\text { torácica y la pelvis?. ¿Cuáles son sL } \\
\text { observar y situar sus puntos visib }\end{array}$ & $\begin{array}{l}\text { rtebran este CIMA 2:. ¿Qué } \\
\text { os puede servir la anatomía } \\
\text { puede servir para nuestro } \\
\text { to?. ¿Cómo funciona la caja } \\
\text { imientos?. ¿Cómo se puede } \\
\text { ore el modelo?. }\end{array}$ \\
\hline
\end{tabular}

Ciclos de Mejora en el Aula (2020). Experiencias de Innovación Docente de la US Esta obra se distribuye con la licencia Creative Commons Reconocimiento-NoComercial-SinObraDerivada 4.0 Internacional (CC BY-NC-ND 4.0.) 


\begin{tabular}{|c|c|c|c|}
\hline \multirow{3}{*}{ S } & \multicolumn{3}{|c|}{ Tiempo utilizado: 60 minutos } \\
\hline & \multicolumn{3}{|c|}{$\begin{array}{l}\text { A continuación iniciamos la práctica del dibujo con los modelos en } \\
\text { vivo y aplicamos todo lo comentado. } \\
\text { Formo grupos de } 4 \text { estudiantes, de manera que dos de ellos } \\
\text { vayan comentando y contrastando sus observaciones frente a los } \\
\text { otros dos (actividad de contraste por pares) y viceversa. Yo voy } \\
\text { de caballete en caballete, comentando errores y aciertos sobre el } \\
\text { dibujo (actividad de contraste), al mismo tiempo que voy heciendo } \\
\text { observaciones teóricas y técnicas de manera general para toda la } \\
\text { clase. Son clases muy activas, con descansos de los modelos. } \\
\text { Tiempo: otros } 60 \text { minutos. }\end{array}$} \\
\hline & \multicolumn{3}{|c|}{ Objetivos: Introducir el tema del CIMA y el problema de partida } \\
\hline \multirow{2}{*}{ P } & $\begin{array}{l}\text { No } 2 \\
(26 / 10 / 2020)\end{array}$ & $\begin{array}{l}\text { Tiempo de taller. } \\
\text { Taller conceptual / } \\
\text { Laboratorio creativo. }\end{array}$ & Tiempo 90 min \\
\hline & \multicolumn{3}{|c|}{$\begin{array}{l}\text { Cada clase (dos horas de duración) cuenta con } 90 \text { minutos posteriores } \\
\text { de tiempo de taller (sin el profesor). } \\
\text { Les entrego a cada alumno una fotocopia con el taller conceptual / } \\
\text { laboratorio creativo, e inician la actividad de taller en grupos pequeños } \\
\text { de } 3 \text { alumnos/as. } \\
\text { Tiempo (1á sesión): } 90 \text { minutos. }\end{array}$} \\
\hline & \multicolumn{3}{|c|}{$\begin{array}{l}\text { Objetivos: Conocer las ideas previas de los alumnos y su capacidad de } \\
\text { investigación y trabajo autónomo. }\end{array}$} \\
\hline
\end{tabular}

\section{Sesión 2: (1a semana) Práctica de clase (dibujo de anatomía artística) y práctica de taller conceptual.}

Grupo 6. Anatomía y Morfología. Facultad de Bellas Artes. US.

\begin{tabular}{|l|l|l|l|}
\hline Modelo & $\begin{array}{l}\text { No } 1 \\
(29 / 10 / 2020)\end{array}$ & $\begin{array}{l}\text { Continuación de la práctica } \\
\text { en clase }\end{array}$ & Tiempo 120 min (2a clase) \\
\hline $\begin{array}{l}\text { Continuan con el planteamiento del problema: la representación } \\
\text { gráfica y reconocimiento morfológico sobre los modelos desnudos } \\
\text { (dibujo del natural) de la Caja torácica (costillas y esternón) y de la } \\
\text { cintura pelviana (huesos ilíacos, sacro y cóccix). Avanzan gráficamente } \\
\text { con el dibujo (soporte papel basic } 70 \times 50 \mathrm{~cm} \text {, grafito). }\end{array}$ \\
\hline
\end{tabular}

Ciclos de Mejora en el Aula (2020). Experiencias de Innovación Docente de la US Esta obra se distribuye con la licencia Creative Commons 


\begin{tabular}{|c|c|c|c|}
\hline \multirow{3}{*}{$A C$} & \multicolumn{3}{|c|}{$\begin{array}{l}\text { Después continuan en los descansos de los modelos con la planificación } \\
\text { y organización práctica del taller conceptual / laboratorio creativo. }\end{array}$} \\
\hline & \multicolumn{3}{|c|}{$\begin{array}{l}\text { A continuación iniciamos la práctica del dibujo con los modelos en } \\
\text { vivo y aplicamos todo lo comentado. } \\
\text { Formo grupos de } 4 \text { estudiantes, de manera que dos de ellos } \\
\text { vayan comentando y contrastando sus observaciones frente a los } \\
\text { otros dos (actividad de contraste por pares) y viceversa. Yo voy } \\
\text { de caballete en caballete, comentando errores y aciertos sobre el } \\
\text { dibujo (actividad de contraste), al mismo tiempo que voy heciendo } \\
\text { observaciones teóricas y técnicas de manera general para toda la } \\
\text { clase. Son clases muy activas, con descansos de los modelos. } \\
\text { Finalizan el dibujo. } \\
\text { Tiempo: } 120 \text { minutos. }\end{array}$} \\
\hline & \multicolumn{3}{|c|}{ Objetivos: Introducir el tema del CIMA y el problema de partida } \\
\hline \multirow{2}{*}{$\begin{array}{l}\text { P } \\
\text { IA } \\
\text { AC }\end{array}$} & $\begin{array}{l}\text { № } 2 \\
(27 / 10 / 2020)\end{array}$ & $\begin{array}{l}\text { Tiempo de taller. Taller } \\
\text { conceptual / Laboratorio } \\
\text { creativo. }\end{array}$ & Tiempo $90 \mathrm{~min}$ \\
\hline & \multicolumn{3}{|c|}{$\begin{array}{l}\text { Cada clase (dos horas de duración) cuenta con } 90 \text { minutos posteriores } \\
\text { de tiempo de taller (sin el profesor). } \\
\text { Les entrego a cada alumno una fotocopia con el taller conceptual } \\
\text { / laboratorio creativo, e inician la actividad de taller en grupos } \\
\text { pequeños de } 3 \text { alumnos/as. } \\
\text { Tiempo (2a sesión): } 90 \text { minutos. }\end{array}$} \\
\hline & $\begin{array}{l}\text { Objetivos: Cor } \\
\text { investigación }\end{array}$ & $\begin{array}{l}\text { cer las ideas previas de lc } \\
\text { trabajo autónomo. }\end{array}$ & alumnos y su capacidad de \\
\hline
\end{tabular}

Sesión 3: Planteamiento del problema, preguntas clave (2a semana) y práctica de taller conceptual. (puesta en práctica del aula abierta / flipped classroom)

Grupo 6. Anatomía y Morfología. Facultad de Bellas Artes. US.

\begin{tabular}{|l|l|l|l} 
Modelo & $\begin{array}{l}\text { No- 1 } \\
(4 / 11 / 2020)\end{array}$ & Planteamiento del problema & $\begin{array}{l}\text { Tiempo } 120 \text { min (1a } \\
\text { clase })\end{array}$ \\
\hline
\end{tabular}

Ciclos de Mejora en el Aula (2020). Experiencias de Innovación Docente de la US Esta obra se distribuye con la licencia Creative Commons Reconocimiento-NoComercial-SinObraDerivada $\quad 4.0$ Internacional (CC BY-NC-ND 4.0.) 

tradicional clase de teoría (magistral). La utilizo para contrastar la información y los proyectos que han ido desarrollando la semana anterior los distintos grupos. Voy de grupo en grupo mientras ellos trabajan.

Tiempo 60 minutos.

La segunda hora es para el inicio de la segunda práctica. Llegan los modelos y damos comienzo al ejercicio. En esta ocasión paso directamente a sondear las ideas previas de los estudiantes. Se IA supone que han visto la clase (vídeo de 30 minutos) que he grabado la semana anterior y colgado en la Plataforma de Enseñanza Virtual (US). Comienzo con el planteamiento del problema: la representación gráfica y reconocimiento morfológico sobre los modelos desnudos (dibujo del natural) de la cintura escapular (escápula y clavícula) y el brazo (húmero, cúbito y radio y mano). Aplico el concepto de aula abierta o (en inglés) flipped classroom.

Repaso con ellos conceptos fundamentales y puntos visibles sobre ambos modelos (masculino y femenino). Les planteo multitud de ejemplos y contraejemplos tanto en los modelos de pose como en los esqueletos de clase, sus formas, y movimientos. Preguntas que vertebran este CIMA 2:. ¿Qué entienden ellos por dibujo?. ¿para qué nos sirve la anatomía y cómo nos afecta en el dibujo?. ¿Nos puede servir para nuestro futuro profesional?. ¿Cómo funciona la cintura escapular, el brazo, antebrazo y mano?. ¿Cuáles son sus movimientos?. ¿Cómo se puede observar y situar sus puntos visibles sobre el modelo?.

A continuación iniciamos la práctica del dibujo con los modelos en vivo y aplicamos todo lo comentado.

Formo grupos de 4 estudiantes, de manera que dos de ellos vayan comentando y contrastando sus observaciones frente a los otros dos (actividad de contraste por pares) y viceversa. Yo voy de caballete en caballete, comentando errores y aciertos sobre el dibujo (actividad de contraste), al mismo tiempo que voy heciendo observaciones teóricas y técnicas de manera general para toda la clase. Son clases muy activas, con descansos de los modelos.

Tiempo: otros 60 minutos.

Objetivos: Introducir el tema del CIMA y el problema de partida

Ciclos de Mejora en el Aula (2020). Experiencias de Innovación Docente de la US Esta obra se distribuye con la licencia Creative Commons 


\begin{tabular}{|c|c|c|c|}
\hline$P$ & $\begin{array}{l}\text { № } 2 \\
(26 / 10 / 2020)\end{array}$ & $\begin{array}{l}\text { Tiempo de taller. Taller } \\
\text { conceptual / Laboratorio } \\
\text { creativo. }\end{array}$ & Tiempo 90 min \\
\hline IA & \multicolumn{3}{|c|}{$\begin{array}{l}\text { Cada clase (dos horas de duración) cuenta con } 90 \text { minutos } \\
\text { posteriores de tiempo de taller (sin el profesor). } \\
\text { Continuan con el taller conceptual / laboratorio creativo, e inician } \\
\text { la actividad de taller en grupos pequeños de } 3 \text { alumnos/as. } \\
\text { Tiempo (3a sesión): } 90 \text { minutos. }\end{array}$} \\
\hline$A C$ & \multicolumn{3}{|c|}{$\begin{array}{l}\text { Objetivos: Conocer las ideas previas de los alumnos y su capacidad } \\
\text { de investigación y trabajo autónomo. }\end{array}$} \\
\hline
\end{tabular}

Sesión 4: (2a semana) Práctica de clase (dibujo de anatomía artística) y práctica de taller conceptual. (puesta en práctica del aula abierta / flipped classroom). Sintesis final, presentación pública y entrega.

Grupo 6. Anatomía y Morfología. Facultad de Bellas Artes. US.

\begin{tabular}{|c|c|c|c|}
\hline 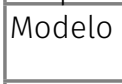 & & & \\
\hline$A C$ & \multicolumn{3}{|c|}{$\begin{array}{l}\text { Es la última sesión de esta segunda práctica de clase. Llegan los } \\
\text { modelos y continuamos el ejercicio. } \\
\text { Aplico el concepto de aula abierta o (en inglés) flipped classroom. } \\
\text { Han visto la clase (vídeo de } 30 \text { minutos) que he grabado la semana } \\
\text { anterior y colgado en la Plataforma de Enseñanza Virtual (US). } \\
\text { Continuamos con el planteamiento del problema: la representación } \\
\text { gráfica y reconocimiento morfológico sobre los modelos desnudos } \\
\text { (dibujo del natural) de la cintura escapular (escápula y clavícula) y } \\
\text { el brazo (húmero, cúbito y radio y mano). } \\
\text { Repaso con ellos conceptos fundamentales y puntos visibles sobre } \\
\text { ambos modelos (masculino y femenino). Les planteo multitud de } \\
\text { ejemplos y contraejemplos tanto en los modelos de pose como } \\
\text { en los esqueletos de clase, sus formas, y movimientos. Preguntas } \\
\text { que vertebran este CIMA 2:. ¿Qué entienden ellos por dibujo?. ¿para } \\
\text { qué nos sirve la anatomía y cómo nos afecta en el dibujo?. ¿Nos } \\
\text { puede servir para nuestro futuro profesional?. ¿Cómo funciona } \\
\text { la cintura escapular, el brazo, antebrazo y mano?. ¿Cuáles son } \\
\text { sus movimientos?. ¿Cómo se puede observar y situar sus puntos } \\
\text { visibles sobre el modelo?. }\end{array}$} \\
\hline
\end{tabular}

Ciclos de Mejora en el Aula (2020). Experiencias de Innovación Docente de la US Esta obra se distribuye con la licencia Creative Commons Reconocimiento-NoComercial-SinObraDerivada 4.0 Internacional (CC BY-NC-ND 4.0.) 
A continuación finalizamos la práctica del dibujo con los modelos en vivo y aplicamos todo lo comentado.

Formo grupos de 4 estudiantes, de manera que dos de ellos vayan comentando y contrastando sus observaciones frente a los otros dos (actividad de contraste por pares) y viceversa. Yo voy de caballete en caballete, comentando errores y aciertos sobre el dibujo (actividad de contraste), al mismo tiempo que voy heciendo observaciones teóricas y técnicas de manera general para toda la clase. Son clases muy activas, con descansos de los modelos.

Vuelvo a entregar en cuestionario inicial (CIMA 2) y les dejo 10 minutos para que lo completen y entreguen.

Tiempo: 120 minutos.

Objetivos: Introducir el tema del CIMA y el problema de partida

\begin{tabular}{l|l|l} 
№ 2 & Tiempo de taller. Taller & Tiempo $90 \mathrm{~min}$
\end{tabular}

$A C$

$(05 / 11 / 2020)$ conceptual / Laboratorio creativo.

Cada clase (dos horas de duración) cuenta con 90 minutos posteriores de tiempo de taller (sin el profesor). Comienza con la S última sesión de la práctica del taller conceptual / laboratorio creativo. La utilizo para contrastar la información y los proyectos que han ido desarrollando la semana anterior los distintos grupos.

Finalizan con el taller conceptual / laboratorio creativo en grupos pequeños de 3 alumnos/as.

Tiempo (4a sesión): 90 minutos.

Objetivos: Conocer las ideas previas de los alumnos y su capacidad de investigación y trabajo autónomo.

Ciclos de Mejora en el Aula (2020). Experiencias de Innovación Docente de la US Esta obra se distribuye con la licencia Creative Commons 
Desarrollo del CIMA 2 / diario de clase.

Como se puede observar en la secuencia de actividades del anterior apartado, diseñé las actividades del CIMA en dos fases diferenciadas en cada clase (dos clases en semana) desarrolladas en dos semanas (26-29 de octubre y 2-5 de noviembre de 2020):

a) 1a Fase (2 horas por clase)

Planteamiento de un problema. Iniciamos la primera clase con una dinámica tradicional. El problema planteado es el dibujo de la caja torácica (costilla y esternón) y de la cintura pélvica sobre los modelos (poses de un modelo masculino y otro femenino). También divido la clase en grupos de 4 alumnos/as para su corrección por pares. Voy de caballete en caballete realizando correcciones, dialogando con ellos, de manera que vean errores y aciertos en sus ejercicios.

\section{b) 2a Fase (Taller conceptual / Laboratorio. 90 minutos por clase) \\ Planteamiento por escrito de 4 problemas de diseño creativo en base a la teoría correspondiente (ANEXO). Esta fase es una introducción totalmente nueva (CIMA) y ajena al programa establecido. Establezco con claridad en el enunciado del ejercicio, las fases del mismo y los grupos formados por tres alumnos/as.}

Siguiendo el anterior esquema, inicié la primera semana (26 a 29 de octubre) la primera fase de este CIMA el lunes 26 de octubre con el grupo 4 (clases lunes de 15 a $17 \mathrm{~h}$ y martes de $18^{\prime} 30$ a 20'30h) con un cuestionario (anexo 1). Son seis preguntas cortas que van en escala ascendente de complejidad. Les dí quince minutos para rellenar y entregar este cuestionario de manera anónima, pero con una referencia para poder reconocer el segundo cuestionario (idéntico al anterior) y comparar la evolución de cada 
alumno y su capacidad de aprendizaje en este breve pero intenso proceso de dos semanas.

El problema inicial de esta primera clase era la de poder dibujar e integrar dentro del modelo la caja torácica y la pelvis. Poses al natural de un modelo masculino y otro femenino en sendas tarimas tradicionales al uso.

La clase magistral la había grabado previamente (programa Kaltura) y colgada dentro de los contenidos del grupo dentro de nuestra Plataforma de Enseñanza Virtual (Blackboard). De esta forma ellos veían la teoría en casa y en el tiempo de clase entramos directamente a realizar la práctica.. Los alumnos podían consultarla desde su casa o desde el móvil (menos de 30 minutos de duración). De esta forma ellos veían la teoría en casa y en el tiempo de clase entramos directamente a realizar la práctica. Con este método ponía en práctica las teorías de aula invertida [1] (en inglés, flipped classroom) .Se inicia la práctica del dibujo de los alumnos $(70 \times 50 \mathrm{~cm})$. Durante las dos horas de duración de esta primera clase, realizan una actividad de contraste mediante la consulta del manual, de la app (skeleton / aplicación gratuita para el teléfono móvil). También divido la clase en grupos de 4 alumnos/as para su corrección por pares. Voy de caballete en caballete realizando correcciones, dialogando con ellos, de manera que vean errores y aciertos en sus ejercicios.

\section{Clase_vídeo:}

https://www.kaltura.com/index.php/extwidget/preview/partner id/2347021/uiconf id/41336841/entry id/1 td5lbmjb/embed/ auto?\&flashvars[leadWithHTML5]=true\&flashvars[sideBarContainer.plugin]=true\&flashvars[sideBarContainer. position]=left\&flashvars[sideBarCont

Antes de iniciar ellos la práctica del dibujo, establezco grupos de cuatro alumnos/as para que entre ellos hagan un ejercicio de corrección organizado por pares. También se descargan y utilizan aplicaciones móviles 
de anatomía, que les facilita la visión correcta del ejercicio práctico de osteología. (https://play.google.com/ store/apps/details?id=com.catfishanimationstudio. SkeletalSystemPreview\&hl=en US\&gl=US).

El tiempo restante de clase (tanto del lunes como el martes) van realizando sus dibujos y voy pasando (de manera muy activa) de caballete en caballete, corrigiendo errores, señalando aciertos y contrastando sus ideas previas.

En la segunda fase introduzco un elemento sorpresa, con la aplicación de un Taller conceptual / laboratorio creativo como nos han mostrado en este curso FIDOP, y que menciona D. Finkel [2]. En nuestra Facultad, tenemos dos horas de clase con el profesor. Tras este tiempo de docencia, los estudiantes tienen 90 minutos de taller. En este tiempo (normalmente con la asistencia de un técnico de taller o con la simple presencia de un alumno interno y sin la impartición de docencia o profesor) los alumnos siguen simplemente trabajando de manera autónoma en la clase. Debo decir, que en el caso de ciertas asignaturas (como es el caso de Anatomía) es habitual que muchos profesores se queden dentro del aula en dicho periodo o en gran parte del mismo. Es un hecho, a pesar de que este tiempo ni contabiliza como docencia ni (en teoría) debería estar presente el profesor. En lugar de continuar con esta "inercia” de prolongación de clase, les planteo el Taller Conceptual (anexo 2). La reacción inicial fue de desconcierto, pero una vez planteado hubo un entusiasmo tremendo y muchas ganas de comenzar el ejercicio en grupos de 3 compañeros. Lo sorprendente es que se quedaban trabajando en el aula incluso en los descansos previstos.

Continuamos con un debate en clase sobre este fenómeno (observar sus conocimientos e ideas previas). Planteamiento del problema (práctica a realizar) con los 
parámetros necesarios. Hipótesis sobre posibles desarrollos tanto en la realidad más cercana y cotidiana como en la realidad paralela (cine de ciencia ficción, vídeo-juegos, etc). En los temas iniciales ya les he dado las herramientas teóricas necesarias sobre los conocimientos básicos, el desarrollo del proceso creativo y la elaboración de un proyecto. Creación de grupos. Elaboración de ideas previas y esquemas de trabajo (entre ellos y posteriormente contrastando la información conmigo). En esta fase iba desplazándome por los diferentes grupos, con una clase muy activa y viendo, comentando y elaborando preguntas en base a su trabajo desarrollado.

En la primera hora de clase de la segunda semana, que está programada (dada la innecesaria presencia de modelos) para la impartición de la clase teórica (clase magistral). Dado que ya la tenían en la Plataforma (vídeo) la utilicé para la continuación del taller conceptual. De esta manera, contamos con una hora extra para esta actividad. La experiencia realizada en el grupo 4 la repetimos con posterioridad con el grupo 6. Este segundo grupo tiene el mismo horario que el anterior (G. 4) pero lo imparto los miércoles y jueves.

\section{Evaluación}

Una vez desarrollada toda la secuencia de actividades de las dos semanas correspondientes al CIMA, debíamos elaborar un método de evaluación del mismo.

Para la evaluación de este CIMA seguí las pautas que se nos plantearon desde este curso: la elaboración de un cuestionario inicial y la repetición del mismo al finalizar el ciclo. Se trataba de comprobar el nivel de conocimiento adquirido por los alumnos y la eficiencia o no de la aplicación de este ciclo en la mejora de su aprendizaje.

Las encuestas debían ser anónimas, pero para poder reconocer la segunda les dije desde el inicio que escribiesen

Ciclos de Mejora en el Aula (2020). Experiencias de Innovación Docente de la US Esta obra se distribuye con la licencia Creative Commons 
un alias o número identificativo y que lo repitiesen en la segunda encuesta. También les comuniqué que aunque no era una actividad con calificación, sí era importante para la evaluación de la actividad.

Los resultados los presento en la siguiente figura (escalera de conocimiento), con los porcentajes de asimilación inicial y final. Me ha centrado en los resultados del grupo 6 , ya que (debido a un caso positivo de covid19) el grupo 4 quedó reducido y tenía menos encuestas de retorno.

Las preguntas que elaboré para este cuestionario fueron:

1) ¿Qué es para ti el dibujo?.¿Para qué sirve el dibujo?

2) ¿Qué es la Anatomía Artística?.¿Piensas que te servirá en tu futuro?.

3) ¿De qué manera piensas que influye nuestro sistema óseo en una pose con modelo?. ¿Podrías nombrar cuatro puntos óseos que se marquen directamente en un modelo humano?.

4) ¿Sabrías explicar la diferencia entre hueso, músculo, tendón y ligamento?.

5) ¿Sabes cuántos tipos genéricos de huesos hay (por su forma)?. ¿Puedes dibujar un ejemplo de cada uno?.

Establecí cinco niveles en las respuestas: A. No sabe / No contesta; B. Conocimiento elemental; C. Conocimiento técnico básico; D. Conocimiento técnico y estético; E. Eficiencia técnica, estética y conceptual. De esta forma obtuve el porcentaje de respuestas reflejada en la gráfica.

En estos momentos creo que el diseño de este cuestionario no ha sido el más interesante una vez concluido todo el ciclo. En cierto modo ahora lo elaboraría de distinta manera. No obstante, el resultado ha sido satisfactorio y ha servido para sondear y conocer los distintos problemas y 
cuestiones que afronta el alumnado al no procesar bien ciertos conceptos básicos. En torno a cinco preguntas encadenadas he procesado el modelo lógico en el dibujo y la anatomía artística en particular, desde conceptos generales fundamentales, hasta particularidades más específicas de la asignatura. Es una herramienta docente muy positiva que permite ver con claridad el conocimiento real e ideas previas de nuestro alumnado.

En la encuesta final introduje una pregunta final para saber cual era su opinión sobre la asignatura y sobre la experiencia específica del CIMA y en concreto con la innovación introducida del taller conceptual / laboratorio creativo. Esta última pregunta me fue muy útil para evaluar la propia experiencia de innovación docente. El resultado fue sorprendente. El $100 \%$ de las encuestas daba por positiva la actividad y en su gran mayoría hacían hincapié en que deberían haber más ejercicios de este tipo en la carrera. Esto lo pude comprobar de manera empírica dado el entusiasmo provocado en ellos la actividad de laboratorio creativo y su frenética actividad durante el desarrollo del mismo. Todos comentaban el crecimiento positivo de su capacidad creativa.

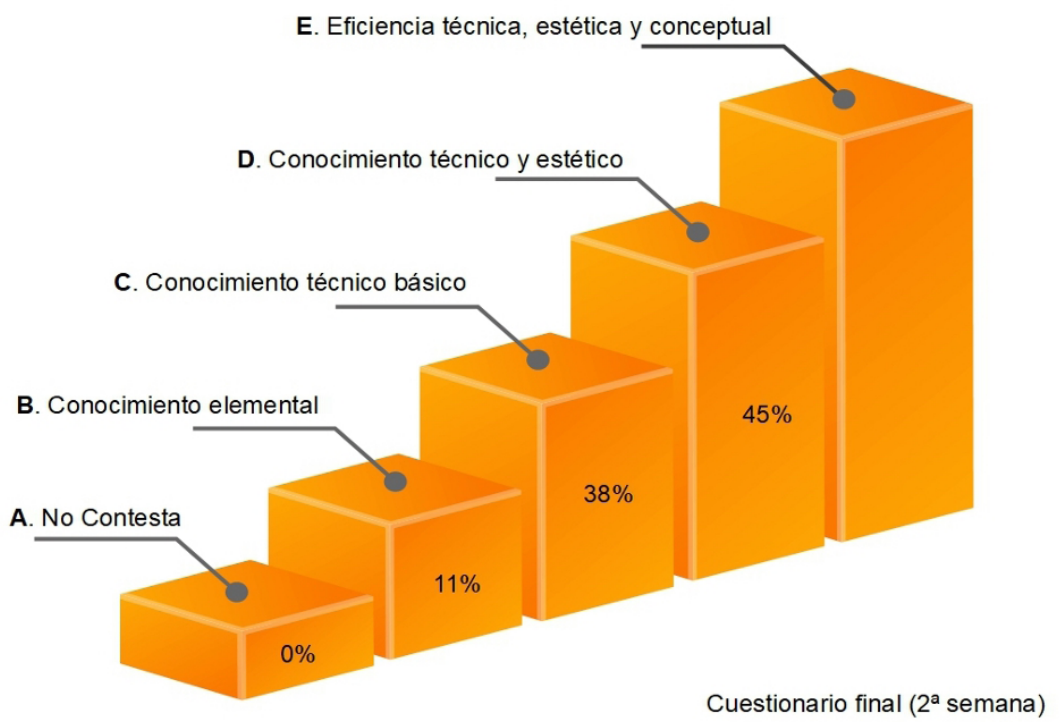

Figura 4. Escalera de conocimiento (a).

Ciclos de Mejora en el Aula (2020). Experiencias de Innovación Docente de la US Esta obra se distribuye con la licencia Creative Commons 


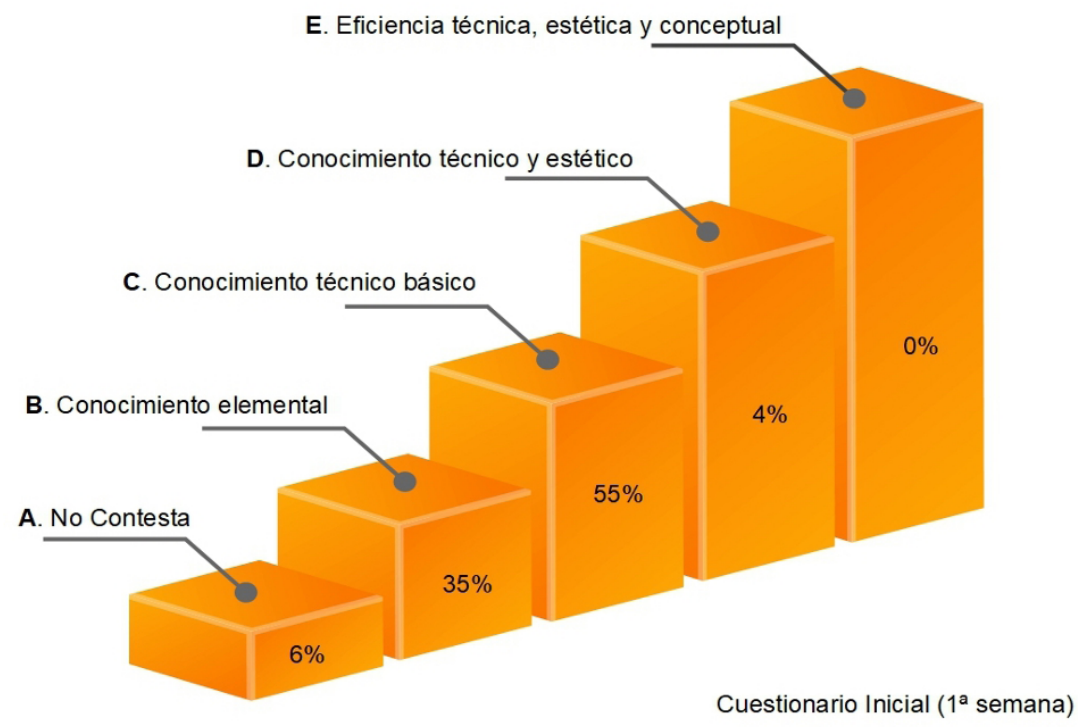

Figura 5. Escalera de conocimiento (b).

\section{Principios didácticos}

Los principios didácticos [3] son los referentes que orientan y guían la actuación del docente (las estrategias) a aplicar en el proceso de enseñanza-aprendizaje (comunicación, personalización, apertura, intuición, globalización, socialización, actividad, creatividad, intuición, etc.). Para el desarrollo de este CIMA me he basado en los siguientes principios didácticos:

1. Conexión con las ideas previas: elaborar estrategias en un nuevo proceso de aprendizaje que permitan una conexión con las ideas previas que posee el alumno, de manera que permita desarrollar una línea de pensamiento lógico y creativo.

2. Mejora de la comunicación: buscar herramientas y métodos que mejoren la comunicación entre el profesor y los alumnos al mismo tiempo que situamos al estudiante en el centro del proceso de aprendizaje.

Ciclos de Mejora en el Aula (2020). Experiencias de Innovación Docente de la US Esta obra se distribuye con la licencia Creative Commons 
3. Actividades para la Motivación: generar actividades que puedan estimular a los alumnos a centrar su atención y despertar su interés por lo que van a aprender.

4. Aprender a Hacer: Los alumnos deben ser capaces de convertir sus conocimientos en instrumentos, para poder estar preparados para la realidad del entorno, tanto en el presente como en el futuro y que adquieran autonomía en su capacidad de investigación.

5. Socialización: El aprendizaje que se transmite a los alumnos debe de penetrar en la vida social de la universidad y en todas las materias académicas, de manera que nos enseñe a convivir con los demás, nos mejore como individuos y como ciudadanos dentro de nuestro ecosistema social, económico y ético.

6. Vinculación de la teoría con la práctica: es importante relacionar el proceso de aprendizaje con el mundo profesional real, que implementa su motivación y facilita su futura inserción.

7. Desarrollo de la creatividad: implementar estrategias que redunden el el idóneo desarrollo de su proceso creativo y del crecimiento de su imaginación.

\section{Conclusiones}

Este ciclo de mejora en el aula me ha supuesto un indudable esfuerzo pero al mismo tiempo una enorme ilusión tras ver los resultados y el entusiasmo que insufló en mis estudiantes. Tras este breve pero intenso proceso, he sacado las siguientes conclusiones:

La primera conclusión es la experiencia positiva al implementar el modelo metodológico descrito en el Ciclo de Mejora. El cambio de paradigma para que el centro de 
atención recaiga sobre el alumno en lugar de ser el profesor. La renovación del concepto tradicional de clase magistral por una pedagogía que implementa la dinámica docente en el aula. Esto no significa excluir la clase magistral del aula, sino generar métodos mixtos que implementen el ejercicio docente y aumente la eficacia del aprendizaje. El inicio fundamental con preguntas clave. El diseño correcto de actividades de contraste que confronten las ideas previas de nuestro alumnado. El estudiante es más consciente de su proceso de aprendizaje. Gana en responsabilidad debido a su autonomía. Facilita la comunicación y la fluidez de conocimiento tanto entre profesor-alumno como entre los propios estudiantes, mejorando y generando una enseñanza mucho más eficiente.

La segunda es que un buen diseño del mapa de contenidos de nuestras asignaturas, nos permite contemplar e identificar cuales de ellos son prioritarios y relacionar contenidos entre sí. Esta herramienta nos facilita la visión de conjunto y la posibilidad de mejora en el proceso de aprendizaje.

La creación de cuestionarios iniciales como herramienta para el conocimiento real y afrontar nuestra enseñanza por los caminos más adecuados. También fomentar la autoevaluación como una herramienta más dentro de su aprendizaje.

Sin duda, la herramienta que me ha resultado de alta eficacia en este ciclo de innovación ha sido la introducción del taller conceptual o laboratorio creativo. Eso ha sido un cambio significativo dentro de la dinámica tradicional de la asignatura. Existe después de cada clase un tiempo expresamente denominado taller, en el que los estudiantes continúan en el aula de manera autónoma el mismo trabajo que se ha realizado en el tiempo de clase. 
El hecho de introducir una actividad específica que además requiere de trabajo en equipo y la inclusión de una alta dosis de creatividad ha sido un revulsivo y un incentivo enorme para ellos. Es una herramienta que seguiré aplicando e implementando en el futuro.

Ciclos de Mejora en el Aula (2020). Experiencias de Innovación Docente de la US (c) (1) E) Esta obra se distribuye con la licencia Creative Commons 
Palabras clave: Anatomía y Morfología, Grado en Bellas Artes, docencia universitaria, experimentación docente universitaria /

Keywords: Anatomy and Morphology, Bachelor of Fine Arts, university teaching, university teaching experimentation.

\section{Referencias bibliográficas}

Finkel, D. (2008). Dar clase con la boca cerrada. Valencia: Universitat de Valencia.

Gomez Hurtado, I. y Garcia Prieto, F.J. (Coord.) (2014). Manual De Didáctica: Aprender A Enseñar. Madrid: Piramide.

Medina Moya, J. L. (2016). La Docencia Universitaria Mediante El Enfoque Del Aula Invertida. Barcelona: Octaedro.

Ciclos de Mejora en el Aula (2020). Experiencias de Innovación Docente de la US Esta obra se distribuye con la licencia Creative Commons 


\section{ANEXO . TALLER CONCEPTUAL / LABORATORIO CREATIVO.}

CIMA 2. FIDOP CURSO GDU 1. 2020. TALLER CONCEPTUAL (Práctica de taller).

Anatomía y Morfología. Grupos 4 y 6 . Profesor Dr. Jesús Algovi González Villegas.

La Práctica (taller conceptual) se realizará en el tiempo de taller durante dos semanas: dos días de clase de 90 minutos cada uno. La clase se organizará por grupos de 3 alumnos/as por proximidad de asiento (dado el escenario actual A por Covid 19). Desarrollo de cada pregunta tanto por escrito como un desarrollo gráfico mediante dibujo u otros medios (TIC).

1) Marta es la hija de 25 años de un multimillonario español. Tiene la enfermedad de "huesos de cristal" (ostogénesis imperfecta). Nos realiza el encargo (bien remunerado) para un pequeño grupo de creadores: el diseño de un exoesqueleto para Marta. Contamos con presupuesto ilimitado de producción e investigación, así como la ayuda interdisciplinar con un equipo de ingenieros mecánicos y médicos ortopedas. Si tenemos éxito se creará una fundación con fondos ilimitados para aplicar estos diseños a aquellas personas con su mismo problema pero sin recursos. ¿Qué diseñarías?.

2) Tras este primer encargo, nos pide reforzarlo, suplementarlo o reformarlo para que impida cualquier tipo de atentado.

3) Nuestro primer encargo es para una persona normal y bípeda. ¿Cómo crees que habríamos evolucionado y desarrollado nuestro estructura ósea si no tuviésemos esqueleto apendicular?. ¿Cómo nos moveríamos?

4) ¿Cómo piensas que sería nuestro esqueleto si nuestra evolución hubiese requerido de levitación o vuelo?. Diseña un exoesqueleto o similar para que Marta pueda levitar o volar. 